\title{
Comparison of the Acute Effects of Acebutolol and Propranolol on Blood Pressure, Heart Rate and Hormonal Changes during Graded Treadmill Exercise in Patients with Essential Hypertension
}

\author{
Hideo MATSUURA, Satoko MASAOKA, Ikuo KANAZAWA, \\ Shigeko MURANO, Yukiko TSUCHIOKA and Goro KAJIYAMA
}

\begin{abstract}
The acute effects of propranolol and acebutolol on blood pressure, heart rate and hormonal changes during graded treadmill exercise were studied in patients with essential hypertension. Both of propranolol ( $2 \mathrm{mg}$ i.v.) and acebutolol (10 $\mathrm{mg}$ i.v.) lowered the pre-exercise hemodynamic parameters and suppressed the elevation of systolic blood pressure, heart rate and pressure-rate product during exercise, but did not show any significant effect on diastolic blood pressure. Although these drugs increased plasma norepinephrine concentration (PNE) at rest and during moderate exercise, they failed to affect PNE at submaximal exercise. Plasma renin activity at rest and during exercise were more strongly suppressed by propranolol than acebutolol. Plasma aldosterone concentration was not affected by these drugs. Propranolol and acebutolol showed similar acute effects on blood pressure, heart rate and hormonal profiles at rest and during exercise within the doses used in this study. These results indicate that $\beta_{1}$ adrenoceptor selectivity and intrinsic sympathomimetic activity may not play an important role in the acute antihypertensive effect at rest and during exercise and that both $\beta$ blockers have beneficial antihypertensive effects during exercise on patients with essential hypertension.
\end{abstract}

Key words: $\quad \beta_{1}$ adrenoceptor selectivity, Intrinsic sympathomimetic activity, Antihypertensive effect

Although the depressor mechanisms of $\beta$ adrenoceptor blocking agents ( $\beta$ blockers) have not been clarified yet $(1,2)$, it is not questionable that $\beta$ blockers are important and useful for the treatment of essential hypertension (3-8). $\beta_{2}$ adrenoceptor blocking action causes increase in peripheral vascular resistance (9-11) and bronchial resistance (12) through the unopposed $\alpha$ stimulation of smooth muscle cells of the vascular and bronchial walls. In order to avoid these adverse effects of $\beta$ blockers, $\beta_{1}$ selective (cardioselective) $\beta$ blockers which have dominant $\beta_{1}$ blocking action compared to $\beta_{2}$ blocking action have been developed (13-16).

The efficacy of antihypertensive drugs have been usually evaluated by their effects on the blood pressure (BP) at rest. On the other hand studies regarding their effects on BP during exercise (17-22) were made to evaluate the usefulness of antihypertensive drugs in daily life, and $\beta$ blockers have been demonstrated to have the potency to lower BP of hypertensive subjects during exercise $(17,18,20$, 21). Comparative studies to evaluate the long term effects of nonselective and cardioselective $\beta$ blockers on hemodynamic and hormonal profiles during dynamic exercise were undertaken using different combinations of these two kinds of $\beta$ blockers (22-27). Only few comparative study, however, has been undertaken between propanolol

From The First Department of Internal Medicine, Hiroshima University School of Medicine, Hiroshima.

Received for publication March 22, 1988.

Reprint request to: Hideo Matsuura, MD, The First Department of Internal Medicine,

Hiroshima University School of Medicine, 1-2-3, Kasumi, Minami-ku, Hiroshima 734, Japan. 
and acebutolol. In many of these studies, exercise was given on a bicycle ergometer but not on a treadmill because of avoiding noise for measurement of blood pressure, and there have been only few acute comparative studies. Since walking is the most popular daily exercise, the acute effects of both kinds of $\beta$ blockers, propranolol and acebutolol, on the hemodynamics and hormonal profiles at rest and during graded treadmill exercise were compared in this study.

\section{SUBJECTS AND METHODS}

Subjects: Thirty-nine patients with essential hypertension (WHO I and II, 6 and 33 patients, respectively) composed of 26 men and 13 women with a mean age of 42.2 years were divided into three groups. Patients were considered to have hypertension if during three subsequent visits without antihypertensive therapy, their sitting blood pressure was more than $160 \mathrm{mmHg}$ in systole and $95 \mathrm{mmHg}$ in diastole. Secondary hypertension was ruled out by clinical, biochemical and radiological examination. The purpose and nature of this study were explained and informed consent was obtained from each patient. Saline was intravenously administered in placebo group (6 men and 4 women, $41.8 \pm 13.7$ years old). Propranolol group (10 men and 5 women, $42.1 \pm 15.2$ years old) and acebutolol group (10 men and 4 women, $42.5 \pm 12.2$ years old) were intravenously administered $2 \mathrm{mg}$ of propranolol and
$10 \mathrm{mg}$ of acebutolol, respectively. No significant differences of blood pressure, heart rate and hormonal backgrounds were observed among these three groups (Table 1).

Treadmill exercise (Fig. 1): Two weeks after discontinuing all antihypertensive medication, treadmill exercise was conducted twice with modified Sheffield's protocol (28) under fasting condition in the morning. The end-point of exercise was $90 \%$ of the predicted maximum heart rate (submaximal exercise). Sixty minutes before the first exercise, an indwelling cannula was inserted into an antecubital vein for blood sampling and telemetric electrocardiogram (ECG) was obtained. They were kept in a supine position for 60 minutes until the beginning of the exercise. BP was measured by the auscultatory

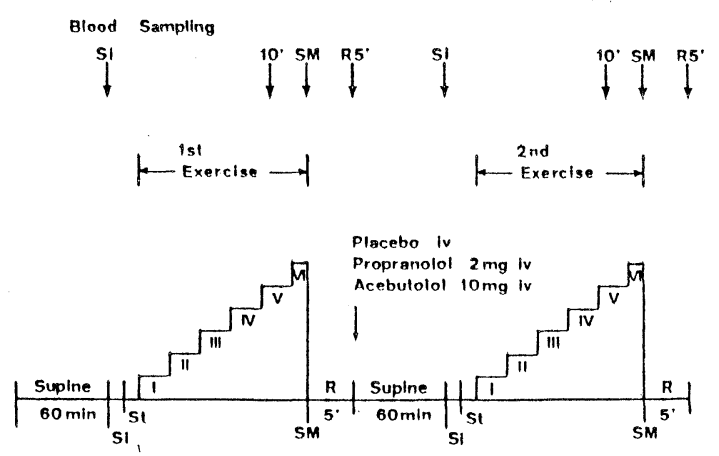

Fig. 1. Study protocol of graded treadmill exercise. $\mathrm{Si}=$ sitting position, $\mathrm{St}=$ standing position, $\mathrm{SM}=$ submaximal exercise, $\mathrm{R}=$ recovery phase.

Table 1. Background of Patients

\begin{tabular}{l|ccc} 
& Placebo & Propranolol & Acebutolol \\
\hline $\mathrm{n}(\mathrm{M} / \mathrm{F})$ & $10(6 / 4)$ & $15(10 / 5)$ & $14(10 / 4)$ \\
WHO I / II & $2 / 8$ & $2 / 13$ & $2 / 12$ \\
age & $41.8 \pm 13.7$ & $42.1 \pm 15.2$ & $42.5 \pm 12.2$ \\
SBP $(\mathrm{mmHg})$ & $150.2 \pm 11.6$ & $154.5 \pm 20.2$ & $157.5 \pm 20.5$ \\
DBP $(\mathrm{mmHg})$ & $97.2 \pm 7.0$ & $101.1 \pm 13.3$ & $102.7 \pm 13.3$ \\
HR $\left(\mathrm{min}^{-1}\right)$ & $75.6 \pm 15.2$ & $72.9 \pm 10.1$ & $74.9 \pm 20.2$ \\
PRP $\left(\times 10^{2} \mathrm{mmHg} \cdot \mathrm{min}^{-1}\right)$ & $113.4 \pm 23.0$ & $112.7 \pm 20.8$ & $118.5 \pm 43.9$ \\
PNE $\left(\mathrm{ng} \cdot \mathrm{ml}^{-1}\right)$ & $0.232 \pm 0.114$ & $0.241 \pm 0.125$ & $0.244 \pm 0.073$ \\
PRA $\left(\mathrm{ng} \cdot \mathrm{ml}^{-1} \mathrm{~h}^{-1}\right)$ & $2.37 \pm 1.09$ & $2.05 \pm 1.52$ & $1.66 \pm 1.06$ \\
PAC $\left(\mathrm{pg} \cdot \mathrm{ml}^{-1}\right)$ & $80.2 \pm 23.9$ & $79.2 \pm 16.3$ & $80.9 \pm 13.0$ \\
Exercise Duration $(\mathrm{min})$ & $13.9 \pm 1.6$ & $14.0 \pm 1.5$ & $14.0 \pm 1.1$ \\
\hline
\end{tabular}

SBP: systolic blood pressure, DBP: diastolic blood pressure, HR: heart rate, PRP: pressure-rate product, PNE: plasma norepinephrine concentration, PRA: plasma renin activity, PAC: plasma aldosterone concentration. 
method using a mercury sphygmomanometer and heart rate (HR) was obtained from ECG recording every minute from 5 minutes before exercise to 5 minutes after exercise. Pressure-rate product (PRP) was calculated as the product of systolic BP and HR. At 5 minutes of recovery, saline as placebo, $2 \mathrm{mg}$ of propranolol or $10 \mathrm{mg}$ of acebutolol was intravenously injected over a period of 10 minutes. Sixty minutes after the patients were kept in a supine position, the second treadmill exercise was performed with exactly the same duration as the first time. Blood samples were drawn just before starting exercise, at 10 minutes of exercise, at the end point of exercise and at 5 minutes of recovery.

Determination of plasma norepinephrine concentration: After plasma was deproteinized by 0.4 $\mathrm{N}$ perchloric acid, norepinephrine (PNE) was absorbed by aluminiumoxide II-III (Merck, Darmstadt, West Germany) at pH 8.4. PNE was determined by the electrochemical method using a high performance liquid chromatography (Yanaco $4000 \mathrm{~W}$, Yanagimoto, Kyoto, Japan) after extracting PNE from aluminiumoxide II-III by $0.01 \mathrm{~N} \mathrm{HCl}$.
Assay of plasma renin activity and plasma aldosterone concentration: Plasma renin activity (PRA) and plasma aldosterone concentration (PAC) were determined by radioimmunoassay using assay kits (CIS, Paris, France).

Statistical analysis: Data were shown as mean \pm SD. The parameters before and after drug administration in each group were compared with the use of Wilcoxon signed rank test. Differences at a $p$ level of less than 0.05 were regarded to be significant.

\section{RESULTS}

1. Backgrounds of the patients in each group (Table 1).

Three groups were statistically alike in regard to age, sex, hemodynamic and hormonal parameters, and duration of the exercise.

2. Effects of the repetitive exercises on blood pressure, heart rate, pressure-rate product and hormonal parameters.

In order to examine the effects of repetitive

Table 2. Effects of repetitive exercise on the hemodynamic and hormonal parameters.

\begin{tabular}{l|r|ccccc} 
& & Si & St & $10 \mathrm{~min}$ & $\mathrm{SM}$ & $\mathrm{R} 5 \mathrm{~min}$ \\
\hline $\mathrm{SBP}$ & 1st & $150.2 \pm 11.6$ & $149.8 \pm 11.5$ & $172.3 \pm 12.9$ & $192.2 \pm 8.6$ & $142.6 \pm 4.9$ \\
$\mathrm{mmHg}$ & 2nd & $146.1 \pm 14.9$ & $143.2 \pm 11.9^{*}$ & $167.9 \pm 16.5$ & $188.4 \pm 13.1$ & $139.6 \pm 12.7$ \\
\hline $\mathrm{DBP}$ & 1st & $97.2 \pm 7.0$ & $95.7 \pm 7.6$ & $96.4 \pm 10.5$ & $96.2 \pm 12.9$ & $93.2 \pm 9.6$ \\
$\mathrm{mmHg}$ & 2nd & $95.1 \pm 8.8$ & $95.0 \pm 10.2$ & $93.6 \pm 9.8$ & $93.0 \pm 14.3$ & $94.0 \pm 8.3$ \\
\hline $\mathrm{HR}$ & 1st & $75.6 \pm 15.2$ & $79.0 \pm 15.9$ & $125.3 \pm 17.5$ & $157.7 \pm 20.1$ & $95.9 \pm 18.1$ \\
$\mathrm{~min}^{-1}$ & 2nd & $79.7 \pm 14.0^{*}$ & $85.0 \pm 17.0^{*}$ & $128.7 \pm 19.1$ & $157.1 \pm 21.4$ & $97.2 \pm 16.1$ \\
\hline $\mathrm{PRP}$ & 1st & $113.4 \pm 23.0$ & $117.9 \pm 25.5$ & $211.9 \pm 38.1$ & $302.2 \pm 30.8$ & $137.2 \pm 30.4$ \\
$\times 10^{2} \mathrm{mmHg}^{-\mathrm{min}^{-1}}$ & 2nt & $116.4 \pm 23.2$ & $121.5 \pm 25.9$ & $216.2 \pm 39.3$ & $294.5 \pm 34.4$ & $135.0 \pm 21.3$ \\
\hline $\mathrm{PNE}$ & 1st & $0.232 \pm 0.114$ & nd & $0.364 \pm 0.148$ & $0.658 \pm 0.303$ & $0.410 \pm 0.133$ \\
$\mathrm{ng} \cdot \mathrm{ml}^{-1}$ & 2nd & $0.257 \pm 0.139$ & nd & $0.386 \pm 0.152$ & $0.670 \pm 0.274$ & $0.403 \pm 0.133$ \\
\hline $\mathrm{PRA}$ & 1st & $2.37 \pm 1.09$ & nd & $3.03 \pm 1.19$ & $4.40 \pm 1.10$ & $4.32 \pm 1.42$ \\
$\mathrm{ng} \cdot \mathrm{ml}^{-1}$ & 2nd & $2.21 \pm 1.09$ & nd & $2.68 \pm 1.21$ & $3.91 \pm 1.34^{*}$ & $4.18 \pm 1.48$ \\
\hline $\mathrm{PAC}$ & 1st & $80.2 \pm 23.9$ & nd & $100.9 \pm 25.2$ & $116.9 \pm 28.1$ & $128.9 \pm 18.7$ \\
$\mathrm{pg} \cdot \mathrm{ml}^{-1}$ & 2nd & $83.2 \pm 15.2$ & nd & $98.7 \pm 13.3$ & $114.3 \pm 14.0$ & $126.1 \pm 25.0$ \\
\hline
\end{tabular}

nd: not determined

$* \mathrm{p}<0.05$ vs the 1 st exercise

Abbreviations used are the same as those shown in Table 1 and Figure 1. 
exercise on the hemodynamic and hormonal parameters before and during exercise, saline was administered to the placebo group. The first exercise did not show any changes in the pre-exercise values for hemodynamic and hormonal parameters before the second exercise, except systolic BP and HR (Table 2). The second exercise produced the same responses in each parameter as during the first exercise, except PRA which showed a significantly lower value $(\mathrm{p}<0.05)$ at the end point of the second exercise.

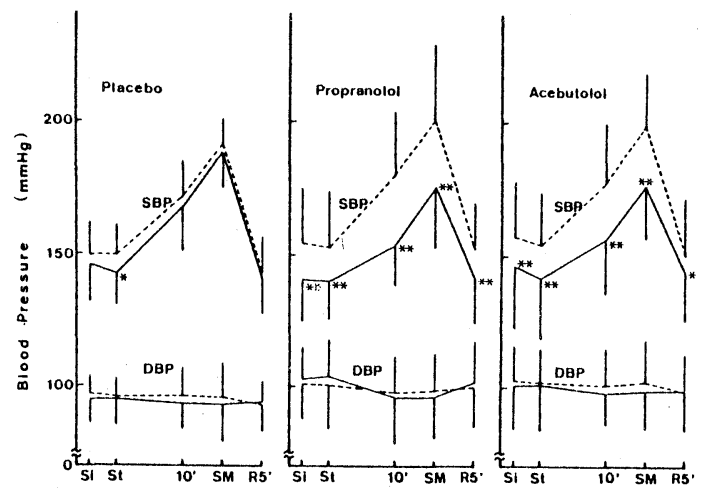

Fig. 2. Effects of placebo $(n=10)$, propranolol $(n=15)$ and acebutolol $(n=14)$ on the changes in blood pressure during exercise. The broken line indicates the time course of parameters before drugs and the solid line indicates that after drugs. Each perpendicular bar indicates the standard deviation. ${ }^{* *} \mathrm{p}<0.01,{ }^{* * *} \mathrm{p}<0.005$ vs before drugs. See Table 1 and Fig. 1 for abbreviations.
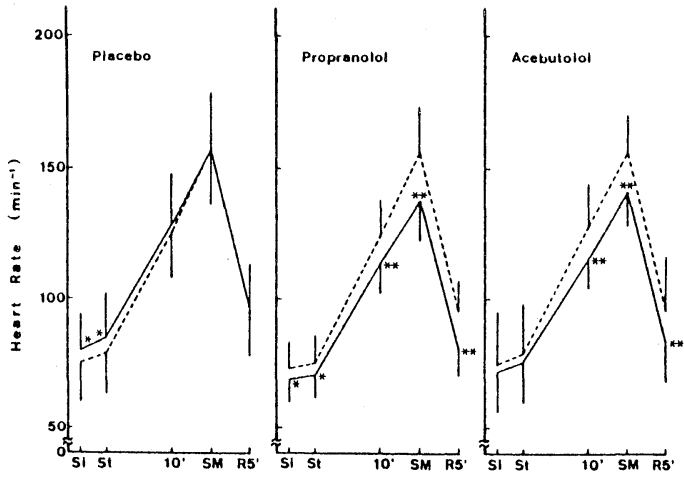

Fig. 3. Effects of placebo $(n=10)$, propranolol $(n=15)$ and acebutolol $(n=14)$ on the changes in heart rate during exercise. ${ }^{*} \mathrm{p}<0.05,{ }^{*} \mathrm{p}<0.01$ vs before drugs. Each perpendicular bar indicates the standard deviation. See Fig. 1 for abbreviations.
3. Effects of propranolol and acebutolol on blood pressure, heart rate and pressure-rate product. Both $\beta$ blockers significantly lowered systolic BP at rest and during exercise, but they failed to lower diastolic BP which showed no change during the exercise (Fig. 2). Propranolol had a more potent depressor effect than acebutolol in the doses used in this study, but there was no statistical significance. In the placebo group, HR was increased by $5 / \mathrm{min}$ 60 minutes after the first exercise $(\mathrm{p}<0.05)$, but propranolol showed a significant decrease in HR at rest $(\mathrm{p}<0.05)$ and acebutolol showed a decreasing tendency. Both $\beta$ blockers significantly suppressed the increase in HR during and after exercise

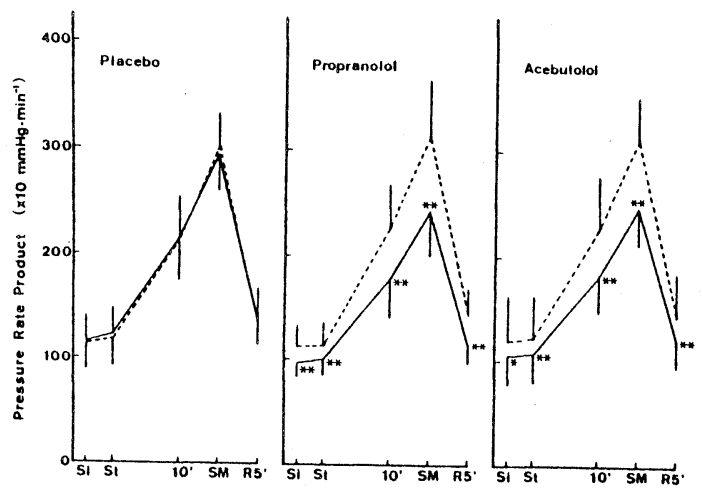

Fig. 4. Effects of placebo $(n=10)$, propranolol $(n=15)$ and acebutolol $(n=14)$ on the changes in pressure-rate product during exercise. Each perpendicular bar indicates the standard deviation. ${ }^{*} \mathrm{p}<0.05,{ }^{*} \mathrm{p}<<0.01$ vs before drugs. See Fig. 1 for abbreviations.

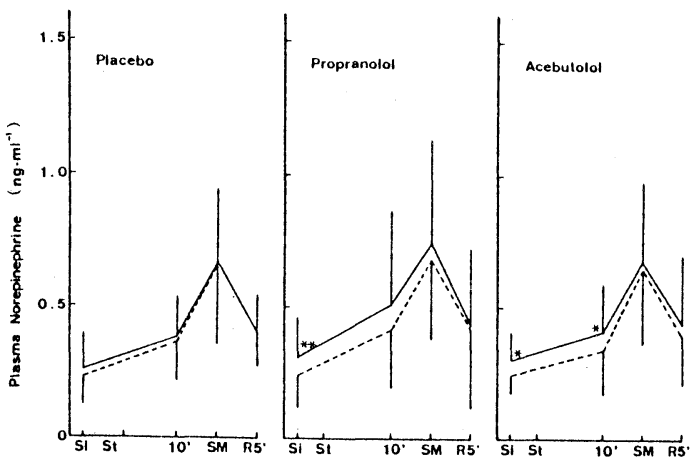

Fig. 5. Effects of placebo $(n=10)$, propranolol $(n=15)$ and acebutolol $(n=14)$ on the changes in plasma norepinephrine concentration during exercise. Each perpendicular bar indicates the standard deviation. ${ }^{*} \mathrm{p}<0.05$, ${ }^{* *} \mathrm{p}<0.01$ vs before drugs. See Fig. 1 for abbreviations. 
( $p<0.01$, Fig. 3). As the results of the changes in $\mathrm{BP}$ and HR by these $\beta$ blockers, PRP was significantly $(\mathrm{p}<0.01)$ decreased at rest and during exercise in both groups (Fig. 4).

4. Effects of propranolol and acebutolol on plasma norepinephrine (Fig. 5).

Propranolol significantly increased PNE before exercise and acebutolol also significantly increased PNE before exercise and at 10 minutes of exercise, but they failed to increase PNE at the end-point of exercise and 5 minutes of recovery.

5. Effects of propranolol and acebutolol on hormonal parameters.

In comparison with the changes in the hemodynamic parameters and PNE, the changes in PRA and PAC during exercise showed a different pattern. They continuously increased until 5 minutes

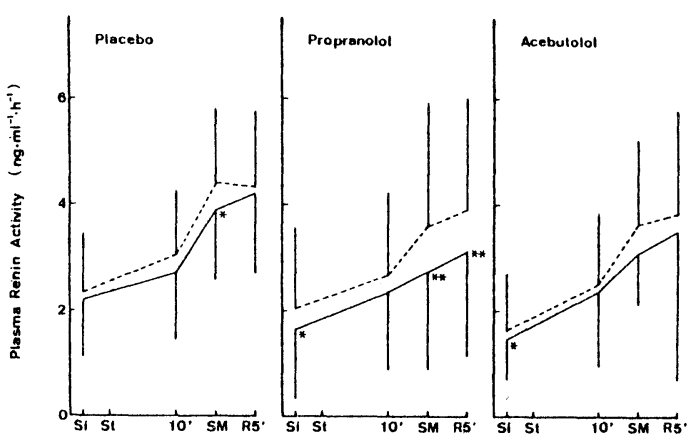

Fig. 6. Effects of placebo $(n=10)$, propranolol $(n=15)$ and acebutolol $(n=14)$ on the changes in plasma renin activity during exercise. Each perpendicular bar indicates the standard deviation. ${ }^{*} p<0.05,{ }^{* *} p<0.01$ vs before drugs. See Fig. 1 for abbreviations.

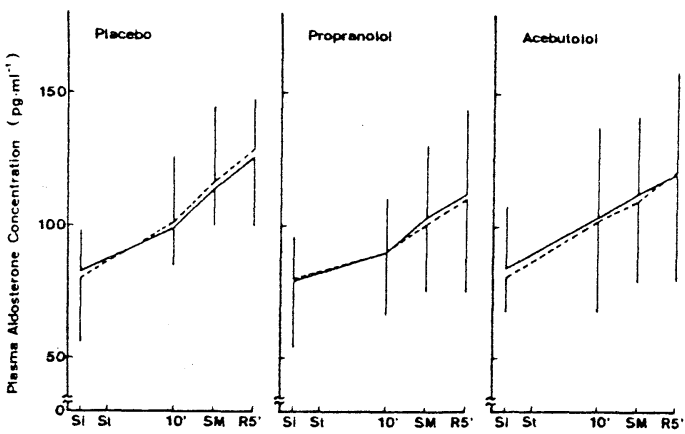

Fig. 7. Effects of placebo $(n=10)$, propranolol $(n=15)$ and acebutol $(n=14)$ on the changes in plasma aldosterone concentration during exercise. Each perpendicular bar indicates the standard deviation. See Fig. 1 for abbreviations. after exercise and returned to the basal level 60 minutes after resting. In the placebo group, PRA after the end-point of the second exercise showed a lower level than that of the first exercise, but there was no statistical difference. Propranolol decreased PRA significantly through the second exercise $(\mathrm{p}<0.05-\mathrm{p}<0.01)$, but acebutolol showed a decreasing tendency in PRA as in the case of placebo (Fig. 6). Although PRA was suppressed by both $\beta$ blockers, the response of PAC to exercise was not affected by them (Fig. 7).

\section{DISCUSSION}

In recent years, many kinds of antihypertensive drugs have been developed and studied pharmacologically and clinically. $\beta$ blockers also have many kinds of derivatives and their modes of action have been thought to be different from each other. They have been classified into five groups by Fitzgerald (29) or three divisions and four groups by Prichard (30) according to their properties. Propranolol has been classified as a $\beta$ blocker with neither partial agonist activity nor cardioselectivity. On the other hand, acebutolol has been classified as a $\beta$ blocker possessing partial agonist activity and cardioselectivity. According to their different properties, effects of these drugs on hemodynamic and hormonal profiles at rest and during exercise may differ. Since hemodynamic and hormonal profiles during exercise are different from those at rest, different types of $\beta$ blockers are considered to show more easily different effects on these profiles during exercise than at rest. Although pindolol and metoprolol $(22,23)$, propranolol and metoprolol (24), propranolol and atenolol $(25,26)$ and nadolol and atenolol (27) were compared, these studies failed to demonstrate different hypotensive effects and negative chronotropic effects between these two kinds of $\beta$ blockers before and during exercise. Kelley (31) also discussed that the effects of cardioselective and noncardioselective $\beta$ blockers on tachycardia during exercise appeared similar.

These previous studies were designed as long term single- or double-blind crossover trials, but our study was designed as an acute open trial. Although propranolol and acebutolol were administered to different groups, the background of patients and the 
changes in all the parameters during the first exercise in each group were not different. This indicates that the design of the present study is good for the purpose of this study.

In studies in which the acute effects of drugs on the parameters during exercise are evaluated, it is important to eliminate the effect of repetitive exercise on the parameters. In other words, the reproducibility of hemodynamic and hormonal parameters should be required at rest and during exercise after the initial exercise. In our previous study, the reproducibility of these parameters was observed in normal subjects (20), and in the current study, this was also observed in patients with essential hypertension. Since the blood pressure, heart rate and hormonal changes were not affected by the repetitive treadmill exercise, the effects of propranolol and acebutolol could be compared.

It was reported that intravenous administration of propranolol failed to lower BP (10) or paradoxically elevate BP (9). During handgrip exercise after intravenous administration of $10 \mathrm{mg}$ of propranolol, elevation of mean arterial BP was not affected, which was due to suppression of cardiac output (CO) and to compensatory increase in total peripheral resistance (TPR) (9). On the other hand, Fleming et al. (17) reported that intravenous administration of propranolol decreased blood pressure due to decrease in cardiac output and no change in peripheral resistance at rest and during exercise. The causes of this discrepancy have not been elucidated, but in this study a significant depressor effect was observed at rest and during exercise after intravenous administration of propranolol and acebutolol. Although CO and TPR were not measured in this study, the depressor effect of these two $\beta$ blockers may be due to decrease in CO. In spite of partial agonist action and cardioselectivity, acebutolol showed the same extent of depressor effect and negative chronotropic effect as propranolol during exercise. These results are compatible with previous studies (22-27).

Franz et al. (22) showed the different effects of pindolol and metoprolol on PNE in hypertensive men. PNE was elevated by metoprolol during submaximal exercise and 5 minutes after exercise, but was not affected by pindolol, except 5 minutes after exercise when norepinephrine was reduced. Kelley
(31) also reported that exercise increased PNE, that the increases were larger in the presence of $\beta$ blockers, and that cardioselective $\beta$ blockers produced larger increases. On the other hand, Esler et al. (32) showed that propranolol affected norepinephrine kinetics, namely inhibition of both norepinephrine spillover and clearance, but failed to affect PNE at rest in patients with essential hypertension. Best et al. (33), however, indicated that infusion of propranolol increased PNE due to inhibition of clearance and no change in spillover of norepinephrine in normal healthy men. Although the precise mechanisms of $\beta$ blockers in affecting PNE in the resting condition (15) have been controversial, propranolol and acebutolol showed similar significant increases in PNE at rest and during mild exercise but not at submaximal exercise or 5 minutes after exercise. This result indicates that cardioselectivity does not affect the increase in PNE by $\beta$ blocking action.

It was reported that PRA was significantly suppressed by $\beta$ blockers (30). Propranolol and acebutolol suppressed the elevation of PRA at rest and during exercise, but acebutolol could suppress PRA less potently than propranolol. Lucsko et al. (34) showed that acebutolol was able to suppress PRA in the supine and standing positions in hypertensives. Weber et al. (35) compared the effects of $\beta$ blockers with different properties and reported that $\beta$ blockers possessing $\beta_{2}$ activity were more prominent in affecting PRA. The present results are in agreement with these previous reports. In spite of the different effects on PRA, propranolol and acebutolol showed no effect on PAC. It may be explained by that aldosterone secretion is controlled by more efficient mechanisms than the renin system. Hansson et al. (19) indicated that long term treatment with penbutolol, possessing intrinsic sympathomimetic activity and membrane stabilizing action, lowered PRA and urinary excretion of aldosterone in hypertensives. Since they did not investigate the acute effects of penbutolol nor the effects on PAC, it is difficult to compare the results in the study.

In summary, two different types of $\beta$ blockers could suppress the elevation of BP during exercise and their hypotensive effects were nearly the same within the dose intravenously administered in this 
study. They also showed the same effect on hormonal changes during exercise except PRA.

ACKNOWLEDGEMENT: The authors are grateful to Miss Yuko Omura for her excellent secretarial assistance.

\section{REFERENCES}

1) Lewis P: The essential action of propranolol in hypertension. Am J Med 60: 837, 1976.

2) Lorimer AR, Dunn FG, Jones JV, et al: Betaadrenoceptor blockade in hypertension. Am J Med 60: 877, 1976.

3) Prichard BNC, Gillan PMS: Use of propranolol (Inderal) in treatment of hypertension. Br Med J 2: 725, 1964.

4) Prichard BNC, Gillan PMS: Propranolol in hypertension. Am J Cardiol 18: 387, 1966.

5) Veterans Administration Cooperative Study Group on Antihypertensive Agents: Propranolol in the treatment of essential hypertension. JAMA 237: 2303, 1977.

6) Veterans Administration Cooperative Study Group on Antihypertensive Agents: Comparison of propranolol and hydrochlorothiazide for the initial treatment of hypertension. I. Results of short-term titration with emphasis on racial differences in response. JAMA 248: 1996, 1982.

7) Veterans Administration Cooperative Study Group on Antihypertensive Agents: Comparison of propranolol and hydrochlorothiazide for the initial treatment of hypertension. II. Results of long-term therapy. JAMA 248: 2004, 1982.

8) Wikstrand J, Westergren G, Berglund G, et al: Antihypertensive treatment with metoprolol or hydrochlorothiazide in patients aged 60 to 75 years. Report from a double-blind international multicenter study. JAMA 255: 1304, 1986.

9) MacDonald HR, Sapru RP, Taylor SH, et al: Effect of intravenous propranolol on the systemic circulatory response to sustained handgrip. Am J Cardiol 18: 333, 1966.

10) Ulrych M, Frohlich ED, Dustan HP, et al: Immediate hemodynamic effects of beta-adrenergic blockade with propranolol in normotensive and hypertensive man. Circulation 37: 411, 1968.

11) Yamamoto J, Sekiya A: On the present action of propranolol in the rat. Arch Int Pharmacodyn 179: 372, 1969.

12) Stephan SA: Unwanted effects of propranolol. Am J Cardiol 18: 463, 1966.

13) Basil B, Jordan R, Loveless AH, et al: Pharmacological properties of M\&B17803A, a cardioselective $\beta$ adrenoceptor blocking agent. J Pharmacol 2: 195, 1971.

14) Ablad B, Carlsson E, Ek L: Pharmacological studies of two new cardioselective adrenergic beta-receptor antagonists. Life Sci 12: 107, 1973.

15) Barrett AM, Carter J, Fitzgerald JP, et al: A new type of cardioselective adrenoceptive blocking drug. Br J Pharmacol 48: 340P, 1973.

16) Harms HH, Spoelstra AJG: Cardiac and bronchial $\beta$ adrenoceptor antagonistic potencies of atenolol, metoprolol, acebutolol, practolol, propranolol and pindolol in the anesthetized dog. Clin Exper Pharmacol Physiol 5: 53, 1978.

17) Shinebourne E, Fleming J, Hamar J: Effects of betaadrenergic blockade during exercise in hypertensive and ischemic heart-disease. Lancet ii: $1217,1976$.

18) Lund-Johansen P: Hemodynamic changes at rest and during exercise in long-term $\beta$-blocker therapy of essential hypertension. Acta Med Scand 195: 117, 1974.

19) Hansson BG, Hokfelt B: Long term treatment of moderate hypertension with penbutolol (Hoe 893d). 1 . Effects on blood pressure, pulse rate, catecholamines in blood and urine, plasma renin activity and urinary aldosterone under basal conditions and following exercise. Europ J Clin Pharmacol 9: 9, 1975.

20) Masaoka S, Kubo S, Kanazawa I, et al: Effects of nifedipine and captopril upon the hemodynamic and hormonal features during treadmill exercise in patients with essential hypertension [Abst]. Jpn Circ J 47: 1003, 1983.

21) Myers MG, De Champlain J: Effects of atenolol and hydrochlorothiazide on blood pressure and plasma catecholamines in essential hypertension. Hypertension 5: 591, 1983.

22) Franz IW, Lohmann FW, Koch G: Differential effects of long-term cardioselective and nonselective betareceptor blockade on plasma catecholamines during and after physical exercise in hypertensive patients. J cardiovasc Pharmacol 2: 35, 1980.

23) Vandongen $R$, Margetts B, Deklerk N, et al: Plasma catecholamines following exercise in hypertensives treated with pindolol: Comparison with placebo and metoprolol. Br J Clin Pharmacol 21: 627, 1986.

24) Morrison SC, Chir B, Kumana CR, et al: Selective and nonselective beta-adrenoceptor blockade in hypertension: Responses to changes in posture, cold and exercise. Circulation 65: 1171, 1982.

25) Leener FHH, Coenen CHM, Zonderland M, et al: Effects of cardioselective and nonselective $\beta$-blockade on dynamic exercise performance in mildly hypertensive men. Clin Pharmacol Ther 28: 12, 1980.

26) Kaiser P, Hylander B, Eliasson K, et al: Effect of betaselective and nonselective beta blockade on blood pressure relative to physical performance in men with systemic hypertension. Am J Cardiol 55: 79D, 1985.

27) Wilcox RG, Hampton JR: Comparison between atenolol and nadolol in essential hypertension at rest and on exercise. Br J Clin Pharmacol 13: 841, 1982.

28) Sheffield LT: Graded exercise test (GXT) for ischemic heart disease. A submaximal test to a target heart rate in exercise testing and training of apparently healthy individuals: A handbook for physicians. Am Heart Assoc, The Committee on Exercise: 35, 1972. 
29) Fitzgerald JP: Perspectives in adrenergic beta-receptor blockade. Clin Pharmacol Ther 10: 292, 1969.

30) Prichard BNC: $\beta$-adrenergic receptor blockade in hypertension, past, present and future. Br J Clin Pharmacol 5: 379, 1978.

31) Kelly JG: Choice of selective versus nonselective beta blockers: Implication for exercise training. Am J Cardiol 55: 162D, 1985.

32) Esler M, Jackman G, Leonard P, et al: Effect of propranolol on noradrenaline kinetics in patients with essential hypertension. Br J Clin Pharmacol 12: 375,
1981.

33) Best JD, Halter JB: Blood pressure and norepinephrine spillover during propranolol infusion in humans. Am J Physiol 248: R400, 1985.

34) Lucsko M, Guedon J: The evaluation of acebutolol in hypertension: Effects on haemodynamics and plasma renin activity. Clin Trials J 3: 86, 1974.

35) Weber MA, Stokes GS, Gain JM: Comparison of the effects of renin release of beta-adrenergic antagonists with differing properties. J Clin Invest 54: 1413, 1974. 